\title{
(Anthracen-9-yl)methyl sulfides as a mechanistic probe for chemical as well as photochemical electron-transfer reactions
}

\author{
Reshma Gopalakrishnan, Jomon P. Jacob, Sa ada Farhana T. Moideen, Ligi M. Lalu, \\ Perupparampil A. Unnikrishnan, and Sreedharan Prathapan*
}

Department of Applied Chemistry, Cochin University of Science and Technology, Cochin-682 022, Kerala, India.

E-mail:prathapans@gmail.com

DOI: $\underline{\text { http://dx.doi.org/10.3998/ark.5550190.p009.341 }}$

\begin{abstract}
We have examined the intermediacy of sulfur-centred radical cations in the one-electron oxidation of (anthracen-9-yl)methyl sulfides. Reaction of (anthracen-9-yl)methyl sulfides with ceric ammonium nitrate (CAN) gave predominantly nitration and oxidation products along with products arising through sulfur-centred radical cations in minor amounts. Upon irradiation, (anthracen-9-yl)methyl sulfides underwent efficient intramolecular single electron transfer leading to a variety of products arising through the intermediacy of sulfur-centred radical cations.
\end{abstract}

Keywords: Single electron transfer, (anthracen-9-yl)methyl sulfides, ceric ammonium nitrate, oxidation, nitration

\section{Introduction}

Electron-transfer reactions in solution have been thoroughly investigated in chemical reaction dynamics and major progress has been made in the field of the dependence of electron-transfer rates on the free energy of reaction, on donor-acceptor distances as well as on the static properties of the solvents. ${ }^{1,2}$ Organic sulfides undergo fast one-electron oxidation reactions, owing to their relatively low ionization potentials. It is reported that organic sulfides are ideal precursors for sulfur-centred radical cations that can be used for probing mechanisms of electron-transfer quenching of excited states as well as for monitoring the fate of sulfur radicals. ${ }^{3-11}$ One of the most frequently used reagents for the oxidative generation of radicals is the strong one-electron oxidant cerium(IV) ammonium nitrate (CAN). CAN has been widely used in organic reactions which include oxidation, ${ }^{12-16}$ oxidative addition, ${ }^{17-21}$ photooxidation, ${ }^{22}$ nitration, ${ }^{23-25}$ deprotection, ${ }^{26,27}$ graft polymerization, ${ }^{28,29}$ etc. Intermediates formed in these reactions may undergo oxidative fragmentation, ${ }^{30,31}$ rearrangement, ${ }^{32-34}$ or $\mathrm{C}-\mathrm{H}, \mathrm{C}-\mathrm{C}$ and $\mathrm{C}-\mathrm{S}$ 
bond $^{35-38}$ cleavages. Organic sulfides can be oxidized with catalytic amounts of $\mathrm{Ce}(\mathrm{IV})$ salts rapidly and selectively to sulfoxides. ${ }^{39,40}$

Anthracene undergoes oxidation with cerium(IV) ammonium nitrate to form the anthracene radical cation which undergoes nitration followed by $\mathrm{O}-\mathrm{N}$ fragmentation and dimerization to give 9,10-anthraquinone, bianthrone etc. ${ }^{41}$ There are also reports on the radical oxidation and addition reactions of some anthracene derivatives with dimethyl malonate in the presence of CAN. ${ }^{42,43}$ In the presence of acetic acid and polyethylene glycol, anthracene reacted with CAN and sodium bromate to give 1-nitro-9,10-anthracenedione. ${ }^{44}$

Several reports discuss photooxidation of organic sulfides under a variety of reaction conditions: (i) autooxidation ${ }^{45-47}$ (ii) electron-transfer sensitization ${ }^{48-50}$ and (iii) singlet oxygen oxidation. ${ }^{51-54}$ Organic sulfides undergo fast one-electron oxidation reactions because of their relatively low ionization potentials. These sulfide radical cations decay through competitive pathways involving deprotonation at $\mathrm{C}_{\alpha}-\mathrm{H}$ bond, C-S fragmentation, oxidation, aromatic substitution and dimerization by photoinduced electron-transfer reaction. ${ }^{55,56}$ (Anthracen-9yl)methyl sulfides with in-built sulfide and arene units are potent candidates for intramolecular electron-transfer reactions leading to intramolecular radical anion/radical cation pairs. In the present study, we examined the reaction of several (anthracen-9-yl)methyl sulfides with CAN in acetonitrile-water and compared these results with photochemical reactions of (anthracen-9yl)methyl sulfides.

\section{Results and Discussion}

Anthracenemethyl sulfides are conveniently synthesized via a one-pot reaction of 9anthracenemethanol, thiourea and the corresponding alkyl halide and also by base promoted onepot reductive coupling of tosylhydrazones with thiols. ${ }^{57,58}$ For the present investigation, we selected six (anthracen-9-yl)methyl sulfides 1a-f (Chart 1) having different steric and electronic environments around the sulfur atom.

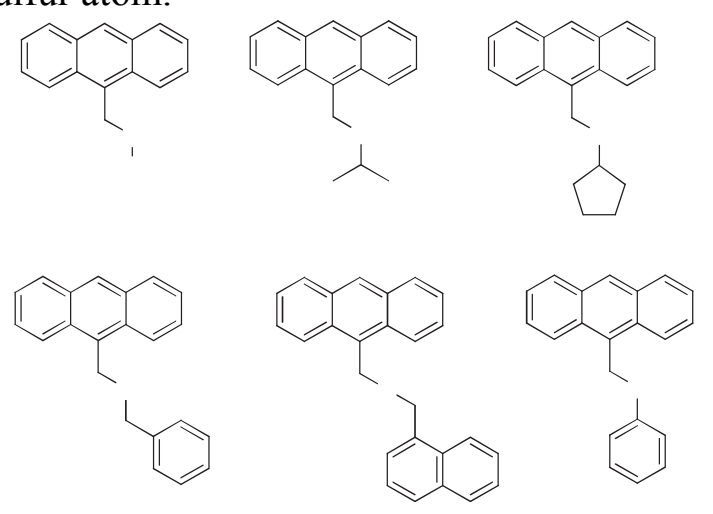

Chart 1. Structures of the (anthracen-9-yl)methyl sulfides. 


\section{CAN-mediated transformations}

When a 1:1 mixture of CAN and (anthracen-9-yl)methyl sulfides 1a-f in 5:1 acetonitrile-water mixture was refluxed for three hours, a variety of products were formed (Scheme 1). Compounds 6 and 7 were formed in major amounts whereas compounds 2-5, 8 and 9 were obtained in low yields.
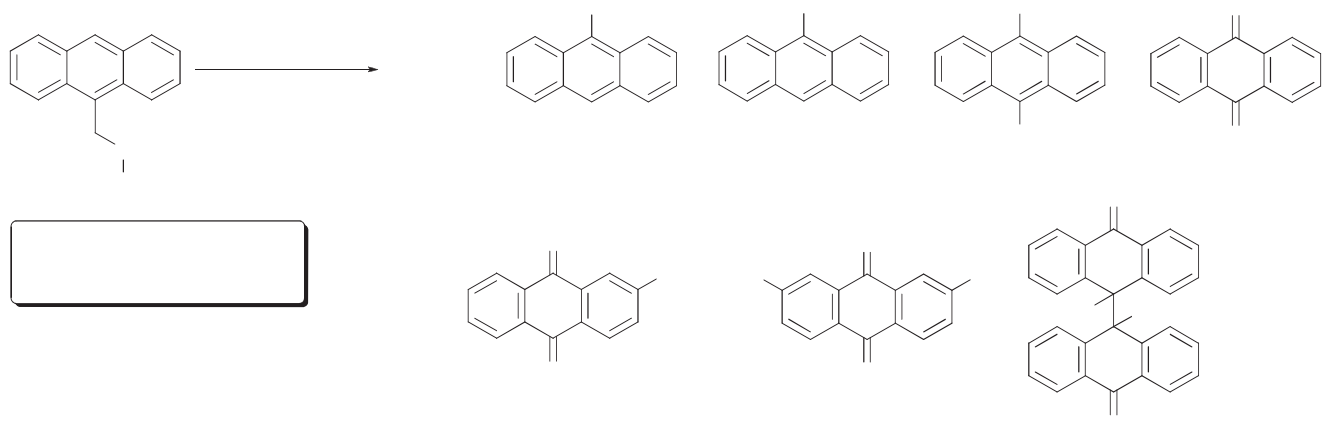

Scheme 1. Products of (anthracen-9-yl)methyl sulfides 1a-f with CAN in acetonitrile-water.

Generation of products 2-9 is explicable on the basis of two distinct reaction pathways. Disulfides 2 and 9-anthracenecarboxaldehyde (3) are generated by sulfur-to-CAN single electron transfer followed by further transformations as detailed in Scheme 2. On the other hand, nitration and oxidation products such as 4-9 are formed by the reaction of CAN with the anthracene component in 1a-f. Among these, formation of 4, 5, 6 and 9 has already been reported in the reaction of anthracenes with CAN. ${ }^{41-44}$ Mechanistic details of CAN-mediated nitration of several polynuclear arenes are available in literature. ${ }^{23,41-44,59-61}$ Interestingly, formation of $\mathbf{7}$ and $\mathbf{8}$ in the CAN-mediated transformation of anthracene and its derivatives has not been reported earlier. A logical conclusion here is that the 9-(2-alkylthiomethyl) substituent plays a persuasive role in the generation of 7 and 8. In order to test this hypothesis, we reexamined the reaction of 9methylanthracene with CAN under identical conditions. Products such as 4-8 were generated in comparable yields in these reactions ruling out any significant role for the 9-(2-alkylthiomethyl) substituent in the generation of $\mathbf{7}$ and $\mathbf{8}$. In order to verify the involvement of the 9-substituent in the formation of $\mathbf{7}$ and $\mathbf{8}$, we repeated the reaction of anthracene with CAN under identical conditions. In contrast to previous reports, products such as $\mathbf{7}$ and $\mathbf{8}$ were formed in substantial amounts in this reaction as well. These results indicated that reactivity of (anthracen-9-yl)methyl sulfides is similar to that of other anthracenes under the conditions employed by us. A major difference here is the concomitant sulfur-to-CAN single electron transfer that takes place as a minor competing pathway for (anthracen-9-yl)methyl sulfides. The nature of the $S$-substituent does not play a significant role in the reaction of (anthracen-9-yl)methyl sulfides with CAN. 


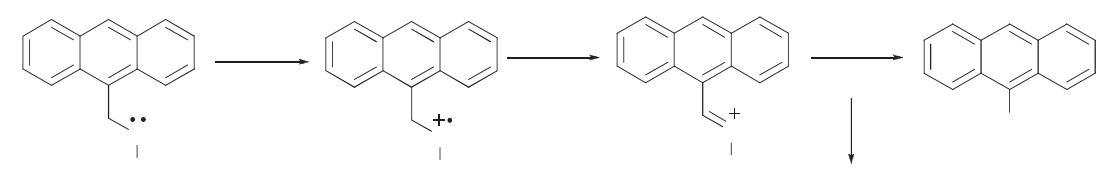

Scheme 2. One electron oxidation of (anthracen-9-yl)methyl sulfides with CAN.

\section{Photoinduced Electron-Transfer Reactions of (Anthracen-9-yl)methyl sulfides}

In order to suppress dimerization via [4+4] addition, all irradiation experiments were conducted at low concentrations. ${ }^{62}$ In a typical run, degassed benzene solutions of (anthracen-9-yl)methyl sulfides 1a-f $(0.8 \mathrm{mM})$ were exhaustively irradiated at $350 \mathrm{~nm}$. After completion of the reaction (45 to 90 minutes), solvent was removed under reduced pressure and the residue was separated by column chromatography. Various fractions were collected and analyzed using spectroscopic measurements. The products formed were identified as 9-anthraldehyde (3), ${ }^{63} 9,10$ anthraquinone (6), ${ }^{64}$ 9-methylanthracene (12), ${ }^{65}$ 1,2-bis(9-anthracenyl)ethane (13), ${ }^{66-69}$ lepidopterene (14), ${ }^{66,70-74}$ biplanene (15), ${ }^{75-77}$ anthrone (16) ${ }^{78}$ along with products arising through the $S$-alkyl residues present in parent anthracenemethyl sulfides. Common products formed in the photoirradiation of (anthracen-9-yl)methyl sulfides 1a-f are shown in Chart 2 and details of all the products isolated are presented in Scheme 3. In the case of 1a, incipient sulfur-containing compounds are volatile and hence escaped detection/isolation. For reasons that are not fully understood, nature of sulfur containing products varies from substrate to substrate.

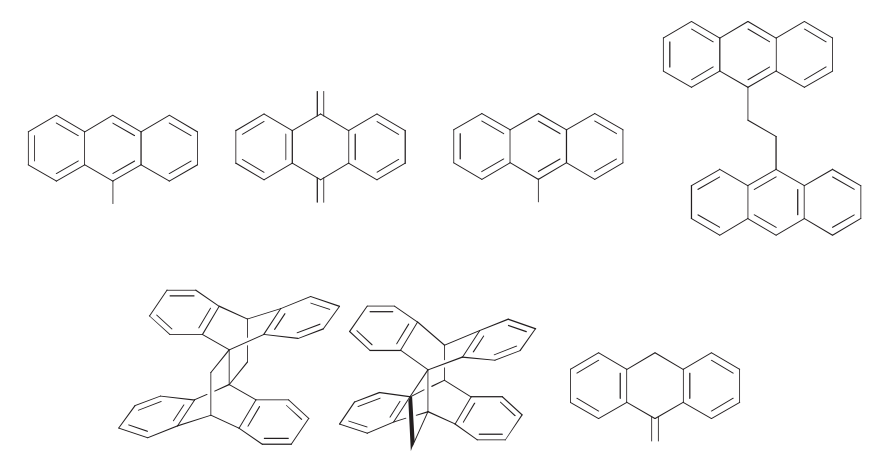

Chart 2. Common products formed in the photolysis of (anthracen-9-yl)methyl sulfides 1a-f. 


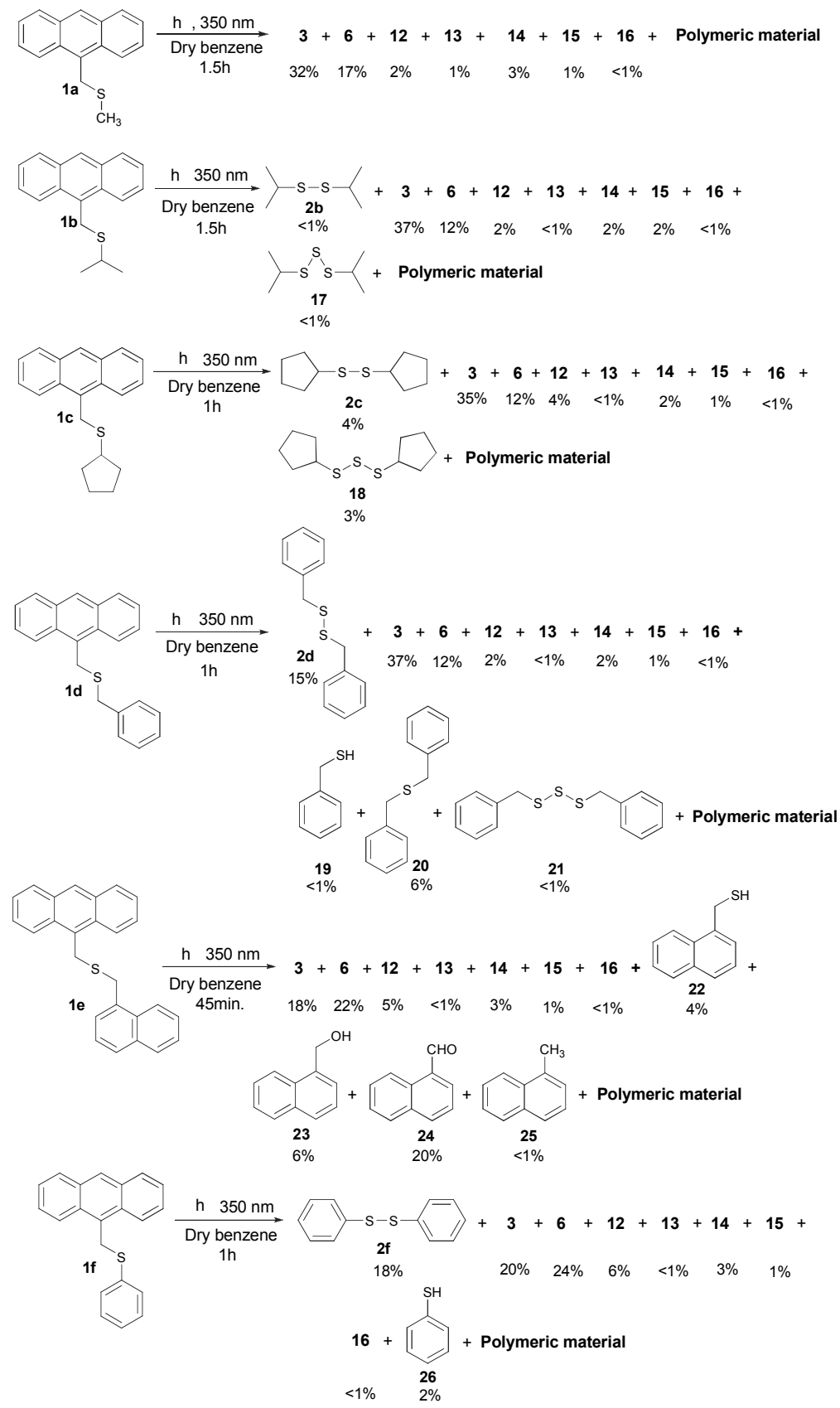

Scheme 3. All reaction products for the photochemical reactions of (anthracen-9-yl)methyl sulfides 1a-f.

Mechanism for the photochemical reaction of (anthracen-9-yl)methyl sulfides 1a-f can be explained on the basis of intramolecular one-electron transfer which takes place in (anthracen-9yl)methyl sulfides in the excited state to form intramolecular sulfide radical cation-anthracene radical anion pair 27. This leads to the destabilization and cleavage of a $\mathrm{C}-\mathrm{S}$ bond to form the anthracenemethyl radical (28) and sulfide radical 29. Sulfide radical (29) undergoes a series of 
reactions to form products such as alcohols, thiols, thioethers, disulfides, and even trisulfides in some cases. The chemistry of the anthracenemethyl radical $(\mathbf{2 8})$ is well documented ${ }^{62,67,73}$ It can be represented as a resonance hybrid of $\mathbf{2 8}(p)$ and $\mathbf{2 8}(\alpha)$ forms. Hydrogen atom abstraction by 28 leads to the formation of 9-methylanthracene (12). Lepidopterene (14) is formed by the $\alpha / p$ dimerization of $\mathbf{2 8}$ followed by intramolecular [4+2] cycloaddition. This is a thermal intramolecular [4+2] cycloaddition, a facile process even at room temperature. ${ }^{79}$ 1,2-Bis(9anthracenyl)ethane (13) is the $\alpha / \alpha$ dimerization product of 28. Biplanene (15) is formed by intramolecular [4+4] cycloaddition of $\mathbf{1 3}$ initiated by stray light. Generation of 9-anthraldehyde (3) is explained on the basis of initial intramolecular excited-state electron transfer leading intermediate 27 followed by $\alpha-\mathrm{H}$ atom loss and bond reorganization to sulfonium cation $\mathbf{3 0}$. Hydrolysis of $\mathbf{3 0}$ gives 9-anthraldehyde (3). 9,10-Anthraquinone (6) is formed through the bond homolysis followed by $\beta$-scission of the endoperoxide 31 generated from $\mathbf{1 a -} \mathbf{f}^{80}$ by the reaction with singlet oxygen ${ }^{81}$ (Scheme 4).

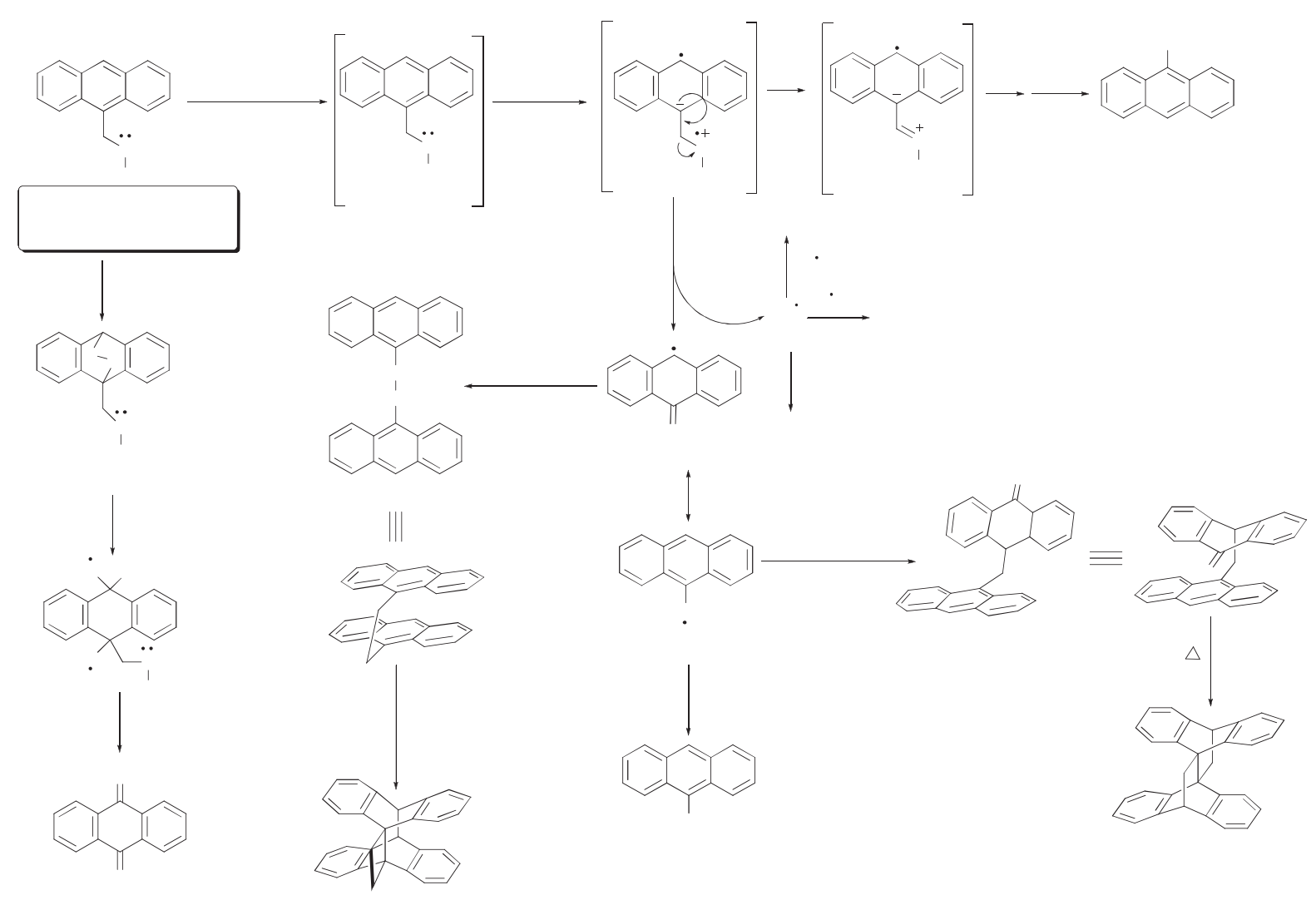

Scheme 4. Proposed mechanistic sequences for the photochemical reactions of (anthracen-9yl)methyl sulfides 1a-f. 


\section{Conclusion}

We have illustrated the single electron-transfer oxidation reaction of (anthracen-9-yl)methyl sulfides via chemical electron transfer as well as intramolecular excited-state electron-transfer methods. We have established that multiple pathways operate in the CAN-mediated and photochemical transformations of anthracenemethyl sulfides. These include single electrontransfer mediated transformations, C-S bond fragmentation, nitration and oxidation as major pathways.

\section{Experimental Section}

General. All reactions were carried out in oven-dried glassware. All experiments used distilled and dried solvents by using standard protocols. All starting materials were purchased from either Sigma-Aldrich or Spectrochem Chemicals and were used without further purification. All the reactions and chromatographic separations were monitored by thin layer chromatography (TLC). Aluminium sheets coated with silica gel (Merck) were used for thin layer chromatography. Separation and purification of compounds was achieved by column chromatography using silica gel (Spectrochem Chemicals, 60-120 mesh). The products were further purified by recrystallization from suitable solvent systems. Melting points are uncorrected and were determined on a Neolab melting point apparatus. Infrared spectra were recorded using Jasco 4100 and ABB Bomem (MB Series) FT-IR spectrometers. The ${ }^{1} \mathrm{H}$ and ${ }^{13} \mathrm{C}$ NMR spectra were recorded at $400 \mathrm{MHz}$ on a Bruker Avance III FT-NMR spectrometer with tetramethylsilane (TMS) as internal standard. Chemical shifts $(\delta)$ are reported in parts per million (ppm) downfield of TMS. Elemental analysis was performed using Elementar Systeme (Vario EL III). Molecular mass was determined by electrospray ionization (ESI) using GC-MS (Agilent GC-7890A, Mass5975C) and fast atom bombardment (FAB) using JMS 600 JEOL mass spectrometers. All new compounds were identified on the basis of spectroscopic and analytical data. Relevant references are cited for known compounds.

\section{Synthesis of (anthracen-9-yl)methyl sulfides.}

(Anthracen-9-yl)methyl sulfides 1a-f were prepared by adaptation of known procedures. ${ }^{57,58,82,83}$

General experimental procedure for the chemical electron-transfer reactions of (anthracen-9-yl)methyl sulfides 1a-f with CAN. To a solution of (anthracen-9-yl)methyl sulfide 1a-f $\left(0.38 \mathrm{~g}, 1\right.$ equiv.) in $\mathrm{MeCN}-\mathrm{H}_{2} \mathrm{O}(5: 1,6 \mathrm{~mL})$, CAN (2.1 equiv.) was added and the mixture was refluxed for $3 \mathrm{~h}$. After completion of the reaction, the reaction mixture was poured into $\mathrm{H}_{2} \mathrm{O}$ and extracted with $\mathrm{CH}_{2} \mathrm{Cl}_{2}$. The organic layer was separated, washed with $\mathrm{H}_{2} \mathrm{O}$ and dried over anhydrous $\mathrm{Na}_{2} \mathrm{SO}_{4}$. Solvent was removed under reduced pressure and the residue was purified by column chromatography on silica gel. Elution with a mixture of hexane and $\mathrm{CH}_{2} \mathrm{Cl}_{2}$ separated twelve products such as compounds 2-9. Compounds $\mathbf{2 b - f}$ were obtained by the elution 
using hexane. Compounds 3-6 were obtained by elution using a mixture of hexane and $\mathrm{CH}_{2} \mathrm{Cl}_{2}$ (3:2). Elution using hexane and $\mathrm{CH}_{2} \mathrm{Cl}_{2}$ (3:7) yielded 7 and 8. Elution using hexane and $\mathrm{CH}_{2} \mathrm{Cl}_{2}$ (1:4) yielded the compound 9.

General experimental procedure for the photoinduced electron-transfer reaction of (anthracen-9-yl)methyl sulfides 1a-f. A degassed solution of (anthracen-9-yl)methyl sulfides 1a-f $(0.8 \mathrm{mM})$ in dry benzene $(200 \mathrm{~mL})$ was irradiated at $350 \mathrm{~nm}$ lamp under argon or nitrogen atmosphere in a Rayonet photochemical reactor. Progress of the reaction was monitored by TLC. Solvent was removed under reduced pressure and the residue was purified by column chromatography over silica gel. Elution with hexane gave $\mathbf{2 b - d , ~ 2 f}$ and $\mathbf{1 2}$. Compounds $\mathbf{1 3}$ and 17-26 were obtained by elution with hexane and $\mathrm{CH}_{2} \mathrm{Cl}_{2}$ (9:1). Compounds $\mathbf{1 4}$ and $\mathbf{1 5}$ are obtained by elution using hexane- $\mathrm{CH}_{2} \mathrm{Cl}_{2}$ (4:1) mixture. Elution with hexane and $\mathrm{CH}_{2} \mathrm{Cl}_{2}(3: 2)$ yielded 3, 6 and 16. The reaction time for different (anthracen-9-yl)methyl sulfides is indicated in Scheme 3. The presence of volatile components was established by GC-MS analysis of the photolysate.

1,2-Diisopropyl disulfide (2b). ${ }^{84,85}$ Colorless liquid; MS: $\mathrm{m} / z 150\left(M^{+}\right)$.

1,2-Dicyclopentyl disulfide (2c). ${ }^{86}$ Colorless liquid; MS: $\mathrm{m} / \mathrm{z} 202\left(M^{+}\right)$.

1,2-Dibenzyl disulfide (2d). ${ }^{87}$ White crystalline solid; mp 67-69 ${ }^{\circ} \mathrm{C}$; MS: $m / z 246\left(M^{+}\right)$.

1,2-bis(Naphthalen-1-ylmethyl)disulfide (2e). ${ }^{88}$ White crystalline solid; mp $145-147{ }^{\circ} \mathrm{C}$; IR $v_{\max }(\mathrm{KBr}): 3092,2986,2883,646,586,479 \mathrm{~cm}^{-1} ;{ }^{1} \mathrm{H}$ NMR $\left(\mathrm{CDCl}_{3}\right): \delta 8.04-7.24(\mathrm{~m}, 14 \mathrm{H}), 4.47$ (s, 4H); MS: $m / z 346\left(M^{+}\right)$; Anal. Calcd for $\mathrm{C}_{22} \mathrm{H}_{18} \mathrm{~S}_{2}$ : C, 76.26; H, 5.24; S, 18.51; Found: C, 76.18; H, 5.17; S, 18.43.

1,2-Diphenyl disulfide (2f). ${ }^{89}$ Colorless crystalline solid; mp 57-59 ${ }^{\circ} \mathrm{C}$; MS: $\mathrm{m} / z 218\left(\mathrm{M}^{+}\right)$.

9-Nitroanthracene (4). ${ }^{90 \mathrm{a}}$ Yellow crystalline solid; mp. 153-157 ${ }^{\circ} \mathrm{C}$; IR $v_{\max }(\mathrm{KBr}): 3056,2923$, 1516, 1317, 1440, $725 \mathrm{~cm}^{-1} ;{ }^{1} \mathrm{H}$ NMR $\left(\mathrm{CDCl}_{3}\right): \delta 8.59-7.56(\mathrm{~m}, 9 \mathrm{H}) ;{ }^{13} \mathrm{C} \mathrm{NMR}\left(\mathrm{CDCI}_{3}\right) \delta 130.9$, 130.4, 128.9, 128.4, 126.2, 122.7, 121.5; MS: $m / z 223\left(M^{+}\right)$; Anal. Calcd for $\mathrm{C}_{14} \mathrm{H}_{9} \mathrm{NO}_{2}$ : C, 75.33; H, 4.06; N, 6.27; Found: C, 75.25; H, 3.98; N, 6.21.

9,10-Dinitroanthracene (5). ${ }^{90 \mathrm{~b}}$ Yellow crystalline solid; mp. 292-294 ${ }^{\circ} \mathrm{C}$; IR $v_{\max }(\mathrm{KBr}): 2955$, 2926, 1540, 1519, 1363, 1283, 831, $768 \mathrm{~cm}^{-1} ;{ }^{1} \mathrm{H}$ NMR $\left(\mathrm{CDCl}_{3}\right): \delta 7.90-7.88(\mathrm{~m}, 4 \mathrm{H}), 7.70-7.68$ (m, 4H); MS: $m / z 268\left(M^{+}\right)$; Anal. Calcd for $\mathrm{C}_{14} \mathrm{H}_{8} \mathrm{~N}_{2} \mathrm{O}_{4}$ : C, 62.69; H, 3.01; N, 10.44; Found: C, $62.60 ; \mathrm{H}, 2.91 ; \mathrm{N}, 10.35$.

2-Nitro-9,10-anthraquinone (7). ${ }^{90 c, e}$ Yellow crystalline solid; mp. $185-186{ }^{\circ} \mathrm{C}$; IR $v_{\max }(\mathrm{KBr})$ : 3060, 1675, 1585, 1525, 1321, 1290, $696 \mathrm{~cm}^{-1} ;{ }^{1} \mathrm{H}$ NMR $\left(\mathrm{CDCl}_{3}\right): \delta 9.06-7.80(\mathrm{~m}, 7 \mathrm{H}) ;{ }^{13} \mathrm{C}$ $\mathrm{NMR}\left(\mathrm{CDCI}_{3}\right) \delta 181.6,181.1,151.3,137.0,134.9,133.3,133.2131 .4,129.2,128.0,127.8$, 127.7, 127.3, 122.7, 120.8; MS: $m / z 253\left(M^{+}\right)$; Anal. Calcd for $\mathrm{C}_{14} \mathrm{H}_{7} \mathrm{NO}_{4}$ : C, 66.41; H, 2.79; N, 5.53; Found: C, 66.36; H, 2.68; N, 5.42.

2,7-Dinitro-9,10-anthraquinone (8). ${ }^{90 \mathrm{~d}}$ Pale yellow crystalline solid; mp $290-291{ }^{\circ} \mathrm{C}$; IR $v_{\max }$ (KBr): 3092, 3038, 1677, 1539, 1356, 1329, 1301, $736 \mathrm{~cm}^{-1} ;{ }^{1} \mathrm{H} \mathrm{NMR}\left(\mathrm{CDCl}_{3}\right): \delta 9.19-8.59(\mathrm{~m}$, $6 \mathrm{H}) ;{ }^{13} \mathrm{C} \mathrm{NMR}\left(\mathrm{CDCI}_{3}\right) \delta 180.24,179.46,151.58,122.83,136.56,134.44,129.64,128.72$; MS: 
$m / z 298\left(M^{+}\right)$; Anal. Calcd for $\mathrm{C}_{14} \mathrm{H}_{6} \mathrm{~N}_{2} \mathrm{O}_{6}$ : C, 56.39; H, 2.03; N, 9.39; Found: C, 56.28; H, 1.94; $\mathrm{N}, 9.32$.

9,9'-Bianthracene-10,10'(9H,9'H)-dione (9). ${ }^{42}$ White crystalline solid; mp 255-256 ${ }^{\circ} \mathrm{C}$; IR $v_{\max }$ (KBr): 3076, 2966, 1675, 1595, 1334, 1288, 811, $690 \mathrm{~cm}^{-1} ;{ }^{1} \mathrm{H}$ NMR $\left(\mathrm{CDCl}_{3}\right): \delta 7.95-6.85(\mathrm{~m}$, $16 \mathrm{H}), 4.77(\mathrm{~s}, 2 \mathrm{H}) ;{ }^{13} \mathrm{C} \mathrm{NMR}\left(\mathrm{CDCl}_{3}\right): \delta 183.0,139.9,133.8,132.2,128.5,127.9,126.7,54.4$; MS: $m / z 386\left(M^{+}\right)$; Anal. Calcd for $\mathrm{C}_{28} \mathrm{H}_{18} \mathrm{O}_{2}$ : C, 87.02; H, 4.69; Found: C, 86.91; H, 4.62.

Anthracen-9(10H)-one (16). ${ }^{91}$ Light yellow crystalline solid; mp 154-155 ${ }^{\circ} \mathrm{C}$; MS: $m / z \quad 194$ $\left(M^{+}\right)$.

1,3-Diisopropyl trisulfide (17). ${ }^{85}$ Colorless liquid; MS: $m / z$ $182\left(M^{+}\right)$.

1,3-Dicyclopentyl trisulfide (18). ${ }^{92}$ Colorless liquid; MS: $\mathrm{m} / \mathrm{z} 234\left(\mathrm{M}^{+}\right)$.

Benzyl thiol (19). ${ }^{93}$ Colorless liquid, MS: $m / z 124\left(M^{+}\right)$.

Dibenzyl sulfide (20).${ }^{94}$ Colorless crystalline solid; mp: $46-48{ }^{\circ} \mathrm{C}$; MS: $\mathrm{m} / z 214\left(M^{+}\right)$.

1,3-Dibenzyl trisulfide (21). ${ }^{95}$ Colorless liquid;MS: $m / z 278\left(M^{+}\right)$.

1-Naphthylmethylthiol (22). ${ }^{96}$ Colorless liquid;MS: $m / z 174\left(M^{+}\right)$.

1-Naphthylmethylalcohol (23). ${ }^{97}$ Colorless liquid;MS: $m / z 158\left(M^{+}\right)$.

1-Naphthaldehyde (24). ${ }^{97}$ Colorless solid; MS: $\mathrm{m} / z 156\left(M^{+}\right)$

1-Methylnaphthalene (25). ${ }^{98}$ Colorless liquid;MS: $m / z 142\left(M^{+}\right)$.

Thiophenol (26). ${ }^{89}$ Colorless liquid, MS: $m / z 110\left(M^{+}\right)$.

\section{Acknowledgements}

We acknowledge STIC, Kochi for NMR and elemental analysis. RG and JPJ gratefully acknowledge UGC, CUSAT and Kerala Government for financial support in the form of Senior Research Fellowships. Funding for this work was provided by DST (PURSE and FIST schemes), UGC (SAP) and Kerala Government.

\section{References}

1. Heitele, H. Angew. Chem., Int. Ed. Engl. 1993, 32, 359. http://dx.doi.org/10.1002/anie.199303591

2. Banu, I. S.; Ramamurthy, P. J. Photochem. Photobiol., A-Chem. 2009, 201, 175. http://dx.doi.org/10.1016/j.jphotochem.2008.10.019

3. Wrzyszczynski, A.; Scigalski, F.; Paczkowski, J. Nukleonika 2000, 45, 73.

4. Chatgilialoglu, C.; Bertrand, M. P.; Ferreri, C. Sulfur-centered radicals in organic synthesis, Alfassi, Z. B. (Eds.); John Wiley \& Sons Ltd; Chichester, U. K. 1999, 311.

5. Bauld, N. L.; Aplin, J. T.; Yueh, W.; Loinaz, A. J. Am. Chem. Soc. 1997, 119, 11381. http://dx.doi.org/10.1021/ja9706965 
6. Lalitha, B.; Mittal, J. $\quad$ P. Radiat. Eff. $1971, \quad 7, \quad 159$. http://dx.doi.org/10.1080/00337577108230982

7. Wolff, R. K.; Aldrich, J. E.; Penner, T. L.; Hunt, J. W. J. Phys. Chem. 1975, 79, 210. http://dx.doi.org/10.1021/j100570a005

8. Bisby, R. H.; Cundall, R. B.; Redpath, J. L.; Adams, G. E. J. Chem. Soc., Faraday Trans. 1 1976, 72, 51 .

http://dx.doi.org/10.1039/f19767200051

9. Chatgilialoglu, C. Sulfur-centered Reactive Intermediates in Chemistry and Biology, Asmus, K. D. (Ed.) Plenum Press: New York, 1990, 116. http://dx.doi.org/10.1007/978-1-4684-5874$\underline{9}$

10. Kosower, N. S.; Kosower, E. M.; Free Radicals in Biology, Pryor, W. A. (Ed.) Academic Press: New York, 1976, 2, 55.

11. Thompson, S. D.; Carroll, D. G.; Watson, F.; O'Donnell, M.; McGlynn, S. P. J. Chem. Phys. 1966, 45, 1367. http://dx.doi.org/10.1063/1.1727769

12. Molander, G. A. Chem. Rev. 1992, 92, 29. http://dx.doi.org/10.1021/cr00009a002

13. Zolfigol, M. A.; Borazjani, M. K.; Mallakpour, S. E.; Nasr-Isfahani, H. Synth. Commun. 2000, 30, 2573. http://dx.doi.org/10.1080/00397910008087422

14. Xiao, J. -P.; Wang, Y. -L.; Jia, X. -S.; Wang, X. -Y.; Wang, H. Synth. Commun. 2000, 30, 1807. http://dx.doi.org/10.1080/00397910008087226

15. Nair, V.; George, T. G.; Nair, L. G.; Panicker, S. B. Tetrahedron Lett. 1999, 40, 1195. http://dx.doi.org/10.1016/S0040-4039(98)02563-5

16. Wang, Y.; Tanko, J. M. J. Chem. Soc., Perkin Trans. 2 1998, 2705. http://dx.doi.org/10.1039/a805655b

17. Nair, V.; Mathew, J.; Prabhakaran, J. Chem. Soc. Rev. 1997, 127.

18. Kobayashi, K.; Tanaka, H.; Tanaka, K.; Yoneda, K.; Morikawa, O.; Konishi, H. Synth. Commun. 2000, 30, 4277. http://dx.doi.org/10.1080/00397910008087050

19. Lee, Y. R.; Kim, B. S.; Kim, D. H. Tetrahedron 2000, 56, 8845. http://dx.doi.org/10.1016/S0040-4020(00)00839-5

20. Nair, V.; Nair, L. G.; Balagopal, L.; Mathew, J. Indian J. Chem. 2000, B39, 352.

21. Hwu, J. R.; King, K. -Y. Curr. Sci. 2001, 81, 1043.

22. Grossi, L.; Strazzari, S. J. Org. Chem. 2000, 65, 2748. http://dx.doi.org/10.1021/jo991856t

23. Mellor, J. M.; Mittoo, S.; Parkes, R.; Millar, R. W. Tetrahedron 2000, 56, 8019. http://dx.doi.org/10.1016/S0040-4020(00)00720-1

24. Hwu, J. R.; Chen, K. -L.; Ananthan, S.; Patel, H. V. Organometallics 1996, 15, 499. http://dx.doi.org/10.1021/om950515c

25. Chakrabarty, M.; Batabyal, A. Synth. Commun. 1996, 24, 1. http://dx.doi.org/10.1080/00397919408012618

26. Hwu, J. R.; Jain, M. L.; Tsai, F. -Y.; Tsay, S. -C.; Balakumar, A.; Hakimelahi, G. H. J. Org. Chem. 2000, 65, 5077. http://dx.doi.org/10.1021/jo000024o 
27. Bull, S. D.; Davies, S. G.; Mulvaney, A. W.; Prasad, R. S.; Smith, A. D.; Fenton, G. Chem. Commun. 2000, 337. http://dx.doi.org/10.1039/b000071j

28. Murugan, R.; Ramakrishna, S. J. Mater. Chem. 2004, 14, 2041. http://dx.doi.org/10.1039/b315169g

29. Jana, S. C.; Maiti, S.; Biswas, S. J. Appl. Polym. Sci. 2000, 78, 1586. http://dx.doi.org/10.1002/1097-4628(20001128)78:9<1586::AID-APP20>3.0.CO;2-9

30. Hwu, J. R.; Shiao, S. -S.; Tsay, S. -C. J. Am. Chem. Soc. 2000, 122, 5899. http://dx.doi.org/10.1021/ja992720f

31. Nair, V.; Panicker, S. B. Tetrahedron Lett. 1999, 40, 563. http://dx.doi.org/10.1016/S00404039(98)02356-9

32. Brimble, M. A.; Nairn, M. R.; Park, J. S. O. J. Chem. Soc., Perkin Trans. $12000,697$. http://dx.doi.org/10.1039/a909243i

33. Schmidt, B.; Wildemann, H. Eur. J. Org. Chem. 2000, 3145. http://dx.doi.org/10.1002/10990690(200009)2000:18<3145::AID-EJOC3145>3.0.CO;2-B

34. Linker, T.; Sommermann, T.; Gimisis, T.; Chatgilialoglu, C. Tetrahedron Lett. 1998, 39, 9637. http://dx.doi.org/10.1016/S0040-4039(98)02264-3

35. Gordon, K. H.; Balasubramanian, S. Org. Lett. 2001, 3, 53. http://dx.doi.org/10.1021/o10067661

36. Guan, X. -P.; Yan, H.; Sun, J. -G.; Yu, Y. -Z. Molecules 1999, 4, 69. http://dx.doi.org/10.3390/40300069

37. Peñéñory, A. B.; Argüello, J. E.; Puiatti, M. Eur. J. Org. Chem. 2005, 114.

38. Capella, L.; Montevecchi, P. C.; Nanni, D. J. Org. Chem. 1994, 59, 7379. http://dx.doi.org/10.1021/jo00103a033

39. Riley, D. P.; Correa, P. E.; J. Chem. Soc., Chem. Commun. 1986, 1097. http://dx.doi.org/10.1039/c39860001097

40. Ali, M. H.; Leach, D. R.; Schmitz, C. E. Synth. Commun. 1998, 28, 2969. http://dx.doi.org/10.1080/00397919808004876

41. Rindone, B.; Scolastico, C. J. Chem. Soc., B 1971, 2238. http://dx.doi.org/10.1039/j29710002238

42. Zengin, M.; Sonmez, F.; Arslan, M.; Kucukislamoglu, M. Maced. J. Chem. Chem. Eng. 2012, 31, 55.

43. Ho, T. -L.; Hall, T. -W.; Wong, C. M. Synthesis 1973, 4, 206. http://dx.doi.org/10.1055/s$\underline{1973-22174}$

44. Hui-qin, Z.; Jin-shun, L.; Feng-xia, Z.; Huaxue Shijie 2007, 487, 428.

45. Sinnreich, D.; Lind, H.; Batzer, H. Tetrahedron Lett. 1976, 3541. http://dx.doi.org/10.1016/S0040-4039(00)71353-0

46. Akasaka, T.; Yabe, A.; Ando, W. J. Am. Chem. Soc. 1987, 109, 8085. http://dx.doi.org/10.1021/ja00260a024

47. Vialaton, D.; Richard, C. J. Photochem. Photobiol., A-Chem. 2000, 136, 169. http://dx.doi.org/10.1016/S1010-6030(00)00344-0 
48. Baciocchi, E.; Giacco, D. T.; Ferrero, M. I.; Rol, C.; Sebastiani, G. V. J. Org. Chem. 1997, 62, 4015. http://dx.doi.org/10.1021/j09615559

49. Baciocchi, E.; Crescenzi, C.; Lanzalunga, O. Tetrahedron 1997, 53, 4469. http://dx.doi.org/10.1016/S0040-4020(97)00119-1

50. Shiraishi, Y.; Taki, Y.; Hirai, T.; Komasawa, I. Chem. Commun. 1998, 2601. http://dx.doi.org/10.1039/a806658b

51. Bonesi, S. M.; Albini, A. J. Org. Chem. 2000, 65, 4532. http://dx.doi.org/10.1021/jo000069p

52. Lacombe, S.; Cardy, H.; Simon, M.; Khoukh, A.; Soumillion, J. -Ph.; Ayadim, M. Photochem. Photobiol. Sci. 2002, 1, 347. http://dx.doi.org/10.1039/b202383k

53. Madhavan, D.; Pitchumani, K. Tetrahedron 2001, 57, 8391. http://dx.doi.org/10.1016/S00404020(01)00834-1

54. Bonesi, S. M.; Fagoni, M.; Monti, S.; Albini, A. Photochem. Photobiol. Sci. 2004, 3, 489. http://dx.doi.org/10.1039/b316891c

55. Giacco, T. D.; Elisei, F.; Lanzalunga, O. Phys. Chem. Chem. Phys. 2000, 2, 1701. http://dx.doi.org/10.1039/a909372i

56. Bonesi, S. M.; Fagnoni, M.; Dondi, D.; Albini, A. Inorg. Chim. Acta 2007, 360, 1230. http://dx.doi.org/10.1016/j.ica.2006.07.022

57. Gopalakrishnan, R.; Jacob, J. P.; Chirakandathil, R.; Unnikrishnan, P. A.; Prathapan, S. Cogent Chemistry 2015, 1 : 1033820. http://dx.doi.org/10.1080/23312009.2015.1033820

58. Eccles, K. S.; Ecloate, C. J.; Lawrence, S. E.; Maguire, A. R. Arkivoc 2010, (9), 216.

59. Hui-qin, Z. Huaxue Shijie 2006, 286, 361.

60. Dinçtürk, S.; Ridd, J. H. J. Chem. Soc., Perkin Trans. 2 1982, 965.

61. Olah, G. A.; Narang, S. C.; Olah, J. A. Proc. Natl. Acad. Sci. 1981, 78, 3298. http://dx.doi.org/10.1073/pnas.78.6.3298

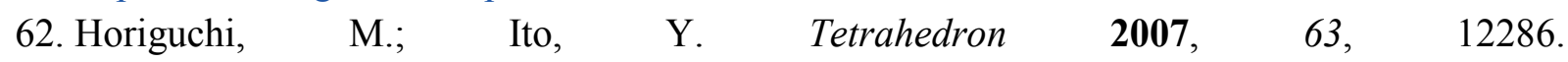
http://dx.doi.org/10.1016/j.tet.2007.09.069

63. Singh, A. K.; Roy, M. Can. J. Chem. 1990, 68, 383. http://dx.doi.org/10.1139/v90-058

64. Tandon, P. K.; Srivastava, M.; Singh, S. B.; Srivastava, N. Synth. Commun. 2008, 38, 3183. http://dx.doi.org/10.1080/00397910802109265

65. Susanto, W.; Chu, C. Y.; Ang, W. J.; Chou, T. C.; Lo, L. C.; Lam, Y. J. Org. Chem. 2012, 77, 2729. http://dx.doi.org/10.1021/jo202482h

66. Vadakkan, J. J.; Mallia, R. R.; Prathapan, S.; Rath, N. P.; Unnikrishnan, P. A. Tetrahedron Lett. 2005, 46, 5919. http://dx.doi.org/10.1016/j.tetlet.2005.06.121

67. Adam, W.; Schneider, K.; Stapper, M.; Steenken, S. J. Am. Chem. Soc. 1997, 119, 3280. http://dx.doi.org/10.1021/ja963643g

68. Higuchi, H.; Otsubo, T.; Ogura, F.; Yamaguchi, H.; Sakata, Y.; Misubi, S. Bull. Chem. Soc. Jpn. 1982, 55, 182. http://dx.doi.org/10.1246/bcsj.55.182

69. Hayashi, T.; Mataga, N.; Sakata, Y.; Misumi, S.; Morita, M.; Tanaka, J. J. Am. Chem. Soc. 1976, 98, 5910. http://dx.doi.org/10.1021/ja00435a027 
70. Deardurff, L. A.; Camaioni, D. M. J. Org. Chem. 1986, 51, 3693. http://dx.doi.org/10.1021/jo00369a024

71. Deardurff, L. A.; Alnajjar, M. S.; Camaioni, D. M. J. Org. Chem. 1986, 51, 3686. http://dx.doi.org/10.1021/jo00369a023

72. Fernandez, M. -J.; Gude, L.; Lorente, A. Tetrahedron Lett. 2001, 42, 891. http://dx.doi.org/10.1016/S0040-4039(00)02151-1

73. Becker, H. -D.; Sandros, K.; Arvidsson, A. J. Org. Chem. 1979, 44, 1336. http://dx.doi.org/10.1021/jo01322a029

74. Duan, S.; Turk, J.; Speigle, J.; Corbin, J.; Masnovi, J.; Baker, R. J. J. Org. Chem. 2000, 65, 3005. http://dx.doi.org/10.1021/j0991495h

75. Vermeersch, G.; Febvay-Garot, N.; Caplain, S.; Couture, A.; Lablache-Combier, A. Tetrahedron Lett. 1979, 20, 609. http://dx.doi.org/10.1016/S0040-4039(01)86015-9

76. Felix, G.; Lapouyade, R.; Castellan, A.; Bouas-Laurent, H.; Gaultier, J.; Hauw, C. Tetrahedron Lett. 1975, 16, 409. http://dx.doi.org/10.1016/S0040-4039(00)71879-X

77. Adam, W.; Schneider, K.; Stapper, M.; Steenken, S. J. Am. Chem. Soc. 1997, 119, 3280. http://dx.doi.org/10.1021/ja963643g

78. Mitsuhashi, T.; Oki, M. Chem. Lett. 1978, 1089. http://dx.doi.org/10.1246/cl.1978.1089

79. Becker, H. -D.; Andersson, K.; Sandros, K. J. Org. Chem. 1980, 45, 4549. http://dx.doi.org/10.1021/jo01311a001

80. Kuroda, S.; Oda, M.; Syumiya, H. Heterocycles 2004, 62, 153. http://dx.doi.org/10.3987/COM-03-S(P)24

81. Danko, M.; Chmela, F.; Hrdlovic, P. Polym. Degrad. Stab. 2003, 79, 333. http://dx.doi.org/10.1016/S0141-3910(02)00297-5

82. Kornblum, N.; Scott, A. J. Org. Chem. 1977, 42, 399. http://dx.doi.org/10.1021/jo00422a069

83. Gopalakrishnan, R.; Jacob, J. P.; Unnikrishnan, P. A.; Prathapan, S. J. Phys. Org. Chem. 2015, 28, 472. http://dx.doi.org/10.1002/poc.3438

84. Rees, C. W.; Rakitin, O. A.; Marcos, C. F.; Torroba, T. J. Org. Chem. 1999, 64, 4376. http://dx.doi.org/10.1021/jo9825005

85. Banfield, S. C.; Omori, A. T.; Leisch, H.; Hudlicky, T. J. Org. Chem. 2007, 72, 4989. http://dx.doi.org/10.1021/jo070099t

86. Hajipoura, A. R.; Ruoho, A. E. Phosphorus, Sulfur, Silicon Relat. Elem. 2003, 178, 1277. http://dx.doi.org/10.1080/10426500307894

87. Oba, M.; Tanaka, K.; Nishiyama, K.; Ando, W. J. Org. Chem. 2011, 76, 4173. http://dx.doi.org/10.1021/jo200496r

88. Tajbakhsh, M.; Lakouraj, M. M.; Mahalli, M. S. Monatsh. Chem. 2008, 139, 1453. http://dx.doi.org/10.1007/s00706-008-0950-0

89. Cha, J. S.; Yu, S. J. Bull. Korean Chem. Soc. 2009, 30, 1588. http://dx.doi.org/10.5012/bkcs.2009.30.7.1588

90. Adams, H.; Bawa, R. A.; McMillan, K. G.; Jones, S. Tetrahedron: Asymmetry 2007, 18, 1003. http://dx.doi.org/10.1016/j.tetasy.2007.04.012 
91. Hadler, H. I.; Raha, C. R. J. Org. Chem. 1957, 22, 433. http://dx.doi.org/10.1021/jo01355a021

92. Balavoine, G.; Barton, D. H. R.; Greed, A.; Lellouche, I. Tetrahedron 1992, 48, 1883. http://dx.doi.org/10.1016/S0040-4020(01)88512-4

93. Han, C. -C.; Balakumar, R. Tetrahedron Lett. 2006, 47, 8255. http://dx.doi.org/10.1016/j.tetlet.2006.09.093

94. Mitsudome, T.; Takahashi, Y.; Mizugaki, T.; Jitsukawa, K.; Kaneda, K. Angew. Chem. Int. Ed. 2014, 53, 8348. http://dx.doi.org/10.1002/anie.201403425

95. Webster, S. A.; Mitchell, S. A.; Gallimore, W. A.; Williams, L. A. D.; Ahmad, M. H. In Vitro Cell. Dev. Biol. Plant. 2008, 44, 112. http://dx.doi.org/10.1007/s11627-008-9125-2

96. Kice, J. L.; Lotey, H. J. Org. Chem. 1989, 54, 3596. http://dx.doi.org/10.1021/jo00276a019

97. Liu, M.; Zhou, F.; Jia, Z.; Li, C.-J. Org. Chem. Front. 2014, 1, 161. http://dx.doi.org/10.1039/c3qo00063j

98. Kealey, S.; Passchier, J.; Huiban, M. Chem. Commun. 2013, 49, 11326. http://dx.doi.org/10.1039/c3cc47203e 